\title{
Multipath Routing Protocols for Mobile Ad Hoc Networks: Security Issues and Performance Evaluation
}

\author{
Rosa Mavropodi and Christos Douligeris* \\ University of Piraeus, Department of Informatics, \\ 80 Karaoli \& Dimitriou, Piraeus 185 34, Greece \\ \{rosa, cdoulig\}@unipi.gr
}

\begin{abstract}
The evolution of wireless network technologies and mobile computing hardware made possible the introduction of various applications in mobile ad hoc networks. These applications have increased requirements regarding security and the acceptable delays in order to provide high quality services. Multipath routing protocols were designed to address these challenges. With the use of multiple paths for the communication between a source and a destination, the autonomic user becomes almost unaware of a possible network failure, due to security attacks or link collapses. Several secure multipath routing protocols have been proposed but little performance information and extensive comparisons are available. In this paper, we briefly describe some security issues that multipath routing protocols face and we evaluate the performance of three existing secure routing protocols under different traffic conditions and mobility patterns.
\end{abstract}

\section{Introduction}

Mobile ad hoc networks have received great attention in recent years, mainly due to the evolution of wireless networking and mobile computing hardware that made possible the introduction of various applications. These applications have increased requirements in order to ensure high quality for the provided services. Security in such infrastructureless networks has been proven to be a challenging task. Multipath routing protocols were initially proposed in order to design robust and secure networks. The maintenance of multiple routes towards a destination prevents initiation of a new path discovery from the source node each time there is a link failure, due to a network fault or a malicious attack. In addition, the existence of multiple paths may prevent node congestion, since it may balances the traffic load through alternative routes.

Routing protocols may generally be categorized as table driven (often called proactive) and source initiated (or on-demand). In table driven protocols

The original version of this chapter was revised: The copyright line was incorrect. This has been corrected. The Erratum to this chapter is available at DOI: 10.1007/978-3-540-32993-0_29

* This work has been supported in part by the IST FET Coordination Action ACCA (006475).

I. Stavrakakis and M. Smirnov (Eds.): WAC 2005, LNCS 3854, pp. 165-176, 2006.

(C) Springer-Verlag Berlin Heidelberg 2006 
(e.g. ZRP [13]), each host continuously maintains complete network routing information. On-demand schemes (e.g. DSR [12]), the routing discovery process is invoked only on demand, in a query/reply approach. According to the number of paths that are discovered from a route request, the routing protocols are divided into single path (e.g. $[12,14])$ and multipath (e.g. $[9,7])$. The number of the discovered paths that are actually used for sending data is another feature of the routing protocols. Some protocols use only a single path for the communication, while others distribute the data through different channels. The route discovery process in the multipath protocols may be initiated either when the active path collapses (in that case communication is performed with one of the alternative paths), or when all known paths towards the destination are broken [5]. The route discovery may stop when a sufficient number of paths are discovered or when all possible paths are detected. The protocols of the second case, are also known as complete. Path found by routing protocols can be node-disjoint [11] or link-disjoint [6] if a node (or a link) cannot participate in more than one route between two end nodes.

The area of secure routing protocols is of particular interest in routing security, since the lack of fixed infrastructure makes routing an obvious target for malicious nodes. Several solutions for secure routing have been proposed, such as collaborative monitoring of the routing behavior between nodes $[15,16]$, motivating nodes to behave well with fictitious currency [18] or participation of nodes in routing paths based on quantifiable criteria [17]. However, these solutions cannot resist Denial-of-Service (DoS) attacks of malicious nodes, since they are designed for single path routing protocols.

Three secure multipath routing protocols have been recently proposed in order to resist Denial of Service (DoS) attacks of collaborating malicious nodes, which single path protocols fail to address; the Secure Routing Protocol (SRP) [8], the multipath routing protocol of [2] and the Secure Multipath Routing protocol (SecMR) [4]. In this paper for simplicity reasons the protocol of [2] will be called Multipath.

In this paper, we evaluate the performance of the currently proposed secure multipath routing protocols of SRP, SecMR and Multipath by simulating their behavior in various traffic scenarios and under different mobility patterns. In section 2, we briefly describe some security issues that routing protocols face in mobile ad hoc networks. In section 3, we present a short description of the compared protocols, while in section 4 we present the simulation results. Finally, in section 5 we discuss possible enhancements and we conclude this paper.

\section{Security Issues in Multipath Routing Protocols}

A major security issue that single path routing protocols fail to resolve is Denial of Service (DoS) attacks of collaborating malicious nodes. With single path routing protocols it is trivial for an adversary to launch a DoS attack, even if security measures are taken. A malicious node controlled by the adversary may participate passively in the routing path between two end nodes and may behave as a legitimate intermediate node. The malicious node can stop the communication at 
any time it seems most advantageous to the adversary. Although communication may be cryptographically protected, network characteristics (such as variation in traffic) or external factors may be used by the adversary in order to identify the proper time to disrupt communication. Even though the end nodes may initiate a new route request after the DoS attack, the time required to establish the new path may be critical. A dedicated and skillful adversary may thus identify the most critical nodes and disable their single routing paths, by compromising a small fraction of nodes.

Multipath routing protocols can be resilient to DoS attacks and may protect network availability from faulty or malicious nodes [1]. Indeed, if there exist $k$ node-disjoint paths between two end nodes, the adversary should compromise at least $k$ nodes - and more particularly at least one node in each path - in order to control their communication. A secure multipath routing protocol must be node-disjoint. In order for a multipath routing protocol to be able to guarantee at a certain level the availability of the communication against DoS attacks of a bounded number $k$ of collaborating malicious nodes, it should employ $k+1$ nodedisjoint routing paths between two communicating nodes. Otherwise, a malicious node would be allowed to participate and consequently control more than one path. Thus, a single malicious node may manipulate the routing protocol and in this way it may compromise all the available routes between two end nodes.

In order to achieve resilience to DoS attacks, a multipath routing protocol should be properly enhanced with cryptographic means, which will guarantee the integrity of a routing path and the authenticity of the participating nodes. However, the cryptographic protection in the route discovery of the secure multipath routing protocols will naturally increase the control overhead. Until now, the efficiency of the secure multipath routing protocols for ad hoc networks has not been thoroughly evaluated.

In order to reduce the control overhead, in several multipath routing protocols e.g. [8], each intermediate node processes each instance of the route request query only the first time it receives it and drops any duplicates. This may lead to discovering less node-disjoint paths from the existing total set of paths between a given source and destination as the second instance of the request is being dropped even if it has propagated through a different neighborhood. This situation is known as the racing phenomenon.

If a protocol requires only end-to end authentication and intermediate nodes participating in a routing path are not authenticated (as for example in SRP), then the protocol is subject to impersonation sybil attacks [19], under which a malicious node may present multiple identities.

\section{Description of the Compared Multipath Routing Protocols}

In this section we will briefly describe the compared routing protocols.

SRP [8] is a routing protocol that manages to find multiple node-disjoint paths. It uses symmetric cryptography in an end-to-end manner, to protect 
the integrity of the route discovery. In SRP the route request message contains unique identifiers, assigned by the source, in order to avoid replay attacks. Each intermediate node will process only the first instance of a route request that it receives and it will drop any other recently heard. When an intermediate node receives the request first checks if it has heard it recently and if not appends itself in the routing path and forwards it, otherwise it drops it. When the target node receives a route request query, the node checks the authenticity of the request by using a symmetric encryption key - a security association - which the two end nodes are supposed to share prior to the request. The route reply query will also be protected with the same security association, in order to protect the integrity of the routing paths. Thus, it is very efficient and it protects from several attacks of malicious nodes. However, the route request propagation is inherently weak to the racing phenomenon, which may prevent the discovery of existing node-disjoint paths. Moreover, the intermediate nodes are not authenticated, making the protocol vulnerable to impersonation and sybil attacks [19]. Thus, a malicious node may participate with fake identities to several paths, rendering the multipath routing insecure.

The secure multipath routing protocol Multipath [2] is based on the FordFulkerson MaxFlow algorithm. In this protocol, when an intermediate node receives a request first checks if a maximum hop distance has been reached. If not appends its neighborhood information along with a signature and forwards the packet, otherwise it drops it. When the target node receives the request, it uses the received information in order to estimate the current network connectivity and to construct the complete set of the existing node-disjoint paths. The protocol exhibits high security characteristics, as all the participating nodes are authenticated and the integrity of the routing path is protected. It manages to find the complete set of the existing node-disjoint paths. However, the propagation of the route request query is not efficient in terms of computation and space costs. The cumulative neighborhood information that the message carries may become larger than the message length. Furthermore, the use of digital signatures by the intermediate nodes of each route request message costs both in delay and processing power and may not be affordable for typically available equipment.

SecMR [4] is a complete secure multipath routing protocol that exhibits authentication in end-to-end and in link-to-link levels and manages to protect the integrity of the routing paths. It works in two phases. The first phase is the neighboring authentication phase which is repeated in periodic time intervals and ensures the link-to-link authentication. During this phase, nodes in range are mutually authenticated through digital signatures. Each node $n_{i}$ constructs a set $N_{i}$ that contains the identifiers of its authenticated neighbors. During the second phase the source produces a signed request, which grants the system with end-to-end authentication, and each intermediate node processes all the receiving requests, ensuring this way that all possible node-disjoint paths will be finally discovered by the destination. When an intermediate node receives a request through a node that belongs to its authenticated list of neighbors, it will first append itself in the routing path. Secondly, it will construct the neigh- 
Table 1. Protocol Comparison

\begin{tabular}{|c|c|c|c|c|}
\hline \multirow[t]{2}{*}{ Characteristics } & \multicolumn{3}{|c|}{ Protocols } & Vulnerabilities \\
\hline & SecMR & Multipath & SRP & $\begin{array}{l}\text { (derived from the luck } \\
\text { of this characteristic) }\end{array}$ \\
\hline end-to-end authentication & yes & yes & yes & luck of data integrity \\
\hline link-to-link authentication & yes & y & no & $\begin{array}{c}\text { impersonation, } \\
\text { sybil attacks }\end{array}$ \\
\hline complete & yes & yes & no & less discovered paths \\
\hline $\begin{array}{c}\text { how many requests } \\
\text { the intermediate node } \\
\text { processes }\end{array}$ & all & all & $\begin{array}{l}\text { only } \\
\text { the } \\
\text { first }\end{array}$ & racing phenomenon \\
\hline
\end{tabular}

borhood information and the exclude-nodes information that are also appended to the message. The neighborhood information will contain all its authenticated neighbors that have not yet received the request and the exclude information will contain all the nodes that have received the message sometime in the past. When a destination receives the request it will check its authenticity by checking its signature, it will construct the node-disjoint paths and will produce a signed reply message, thus protecting the integrity of the used path. Table 1 briefly presents the comparison issues that were discussed in this section.

\section{Performance Evaluation}

Our study involves a comparison of the route request query between SRP, Multipath [2] and SecMR [4] protocols. We implemented the simulator within the NS-2 library. Our simulation modeled a network of 50 hosts placed randomly within a $1500 \times 1000 \mathrm{~m}^{2}$ area. Each node has a radio propagation range of 250 meters and channel capacity was $2 \mathrm{Mb} / \mathrm{s}$.

The nodes in the simulation move according to the 'random way point' model. At the start of the simulation, each node waits for a pause time, then randomly selects and moves towards a destination with a speed uniformly lying between zero and the maximum speed. On reaching this destination it pauses again and repeats the above procedure till the end of the simulation. The minimum and maximum speed is set to 0 and $20 \mathrm{~m} / \mathrm{s}$, respectively and pause times $0,5,10,20,30$ and $40 \mathrm{sec}$. A pause time of $0 \mathrm{sec}$ corresponds to the continuous motion of the node and a pause time of $40 \mathrm{sec}$ corresponds to the time that the node is stationary.

Ten traffic generators were developed to simulate constant bit rate (CBR) sources. Each source generates data packet continuously until the end of the simulation run. The sources and the destinations are randomly selected with uniform probabilities. The size of the data payload was 512 bytes. Each run is executed for $350 \mathrm{sec}$ of simulation time. We used the IEEE 802.11 Distributed Coordination Function (DCF) as the medium access control protocol. The destination of the traffic waits, if necessary, for 5 seconds until it assumes that all possible paths have been found, selects the node-disjoint ones and generate Reply messages concerning these paths. We generated various traffic scenarios by 
using the interarrival data packet time. For each traffic scenario, ten different movement patterns were used.

A free space propagation model with a threshold cutoff was used in our experiments. In the radio model, we assumed the ability of a radio to lock onto a sufficiently strong signal in the presence of interfering signals, i.e., radio capture.

In order to compare the performance of the three routing protocols we evaluated them with respect to the following metrics.

Average end-to-end delay or mean overall packet latency: It is the average delay a packet suffers from the time it leaves the sender application and the time it arrives at the receiver.

Destination location time: Is the average time taken for the first instance of a route request to reach the target node (destination).

Request Propagation time: It corresponds to the average total time that a route request message takes to propagate through the entire network. Thus it is an important metric as it can describe the burden that the request process puts into the entire network. In comparison with the Destination location time metric, the request propagation time illustrates the time that a request zombies in the network and it is processed by nodes that are not going to participate in the path for a given source and destination.

Drop percentage: The percentage of the packets that are dropped due to various reasons.

Routing throughput: The throughput of the routing control packets in the entire the network, averaged by the total number of nodes.

Figure 1 shows the average delay of the received data packets per data interarrival time and pause time 20 secs. We can observe from the results that both SRP and SecMR outperform Multipath even when the interarrival time is small, which depicts high traffic conditions. In both SRP and SecMR the number of generated messages during the route discovery process are kept in sufficiently low levels while the ones of Multipath tend to flood the network. This happens because in Multipath, each intermediate node forwards all the route requests that reaches it for a given source, destination and sequence number, while SRP forwards only the first and SecMR performs a selective forward with the use of the exclude list. This flooding of the network results in higher delay in the data packet delivery. Figure 2, which presents the average delay of the received data packets to a network that transmits 100 data packets per second per with pause time, strengthens the above observations. Indeed, as shown, the SecMR and SRP protocols handle high mobility conditions better, although with a larger pause time the behavior of Multipath tends to converge to the performance of the other two protocols.

Figure 3 presents the dropping percentage of the data packets in relation to interarrival times and a pause time of 20 secs. All three protocols exhibit comparable performance, but SecMR and SRP manage to drop less packets, especially as the interarrival time is getting larger. The observed high drop- 


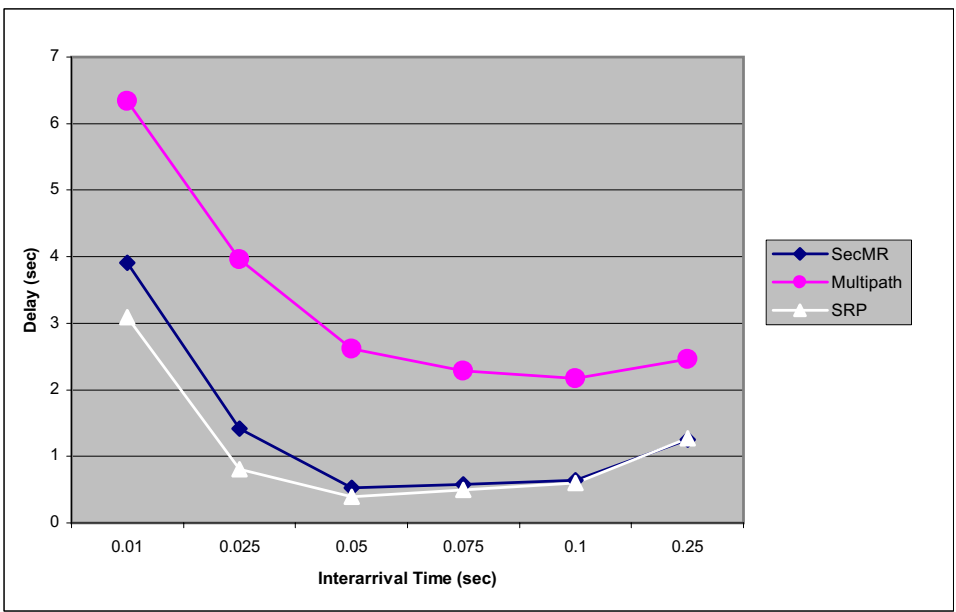

Fig. 1. Average end-to-end data packet delay per interarrival time

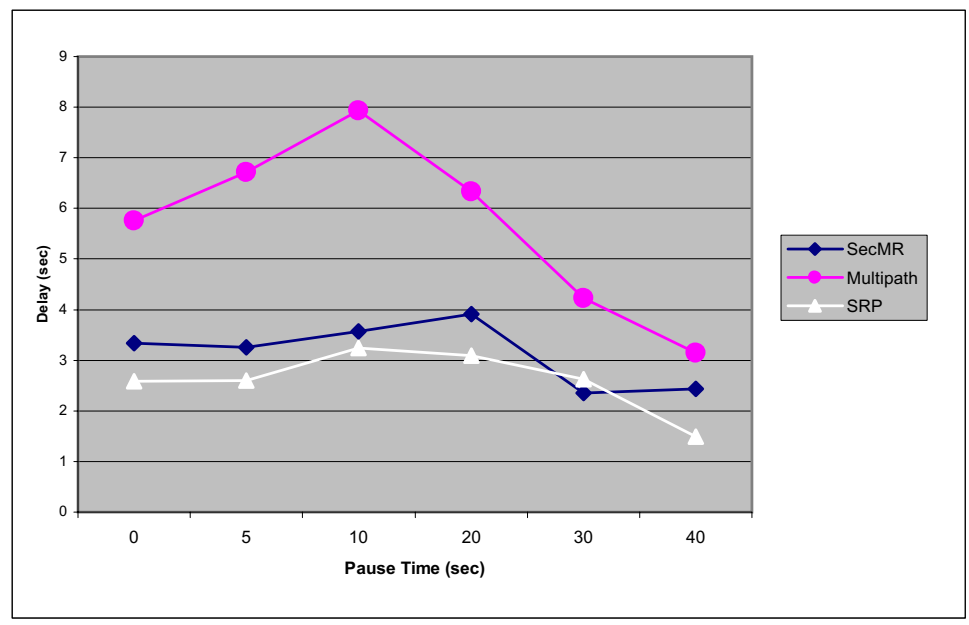

Fig. 2. Average end-to-end data packet delay per pause time

ping ratio, that all three protocols present is mainly due to the configuration of the simulation, namely the expiration time of the paths in the routing tables. Nevertheless, the performance pattern reveals the better performance of SecMR and SRP. Figure 4 shows the dropping percentage of the data packets as this evolves in comparison to various pause times with a data interarrival of 0.25 secs. As figure 4 shows the protocols manage to preserve their dropping pattern under different mobility conditions.

The number of packets that are correctly received by the destination node per interarrival time and a pause time of 20 secs are shown in figure 5 . The performance of all three protocols tends to converge as the interarrival time is 


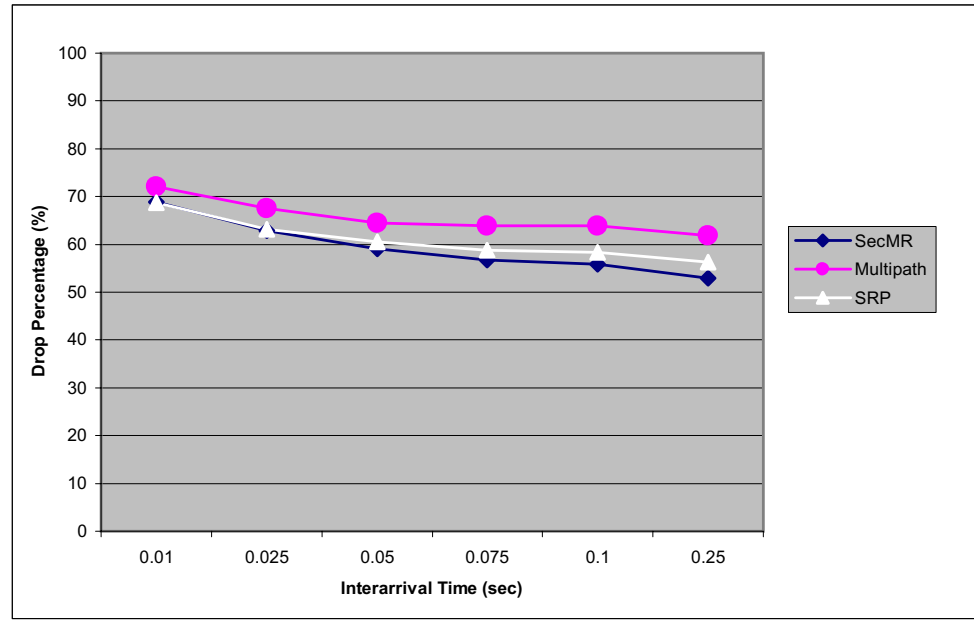

Fig. 3. Data packet drop percentage per interarrival time

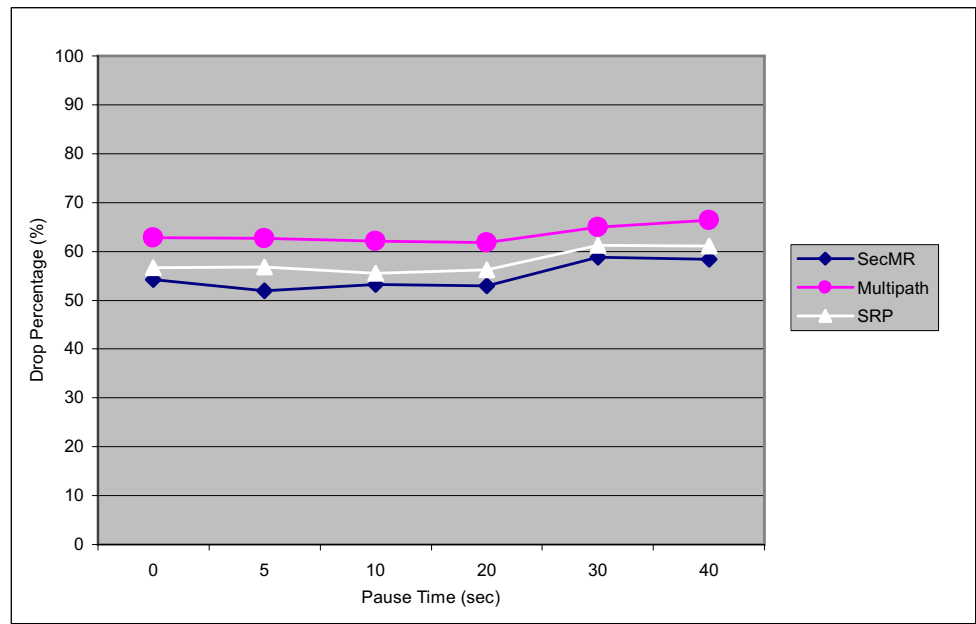

Fig. 4. Data packet drop percentage per pause time

getting larger, that is because in this case the network is facing looser traffic conditions. As one can see SRP and SecMR manage to serve more packets in comparison to Multipath. That is mainly due to the fact that data packets in Multipath encounter higher delay during their propagation and higher dropping rate. All three protocols manage to maintain their behavior with regard to the message delivery ratio under various movability patterns, as shown in figure 6 , which represent a data packet interarrival time of 0.01 secs.

Figure 7 presents the average total time that route request messages propagate through the network. In Multipath, as nodes are getting more and more 


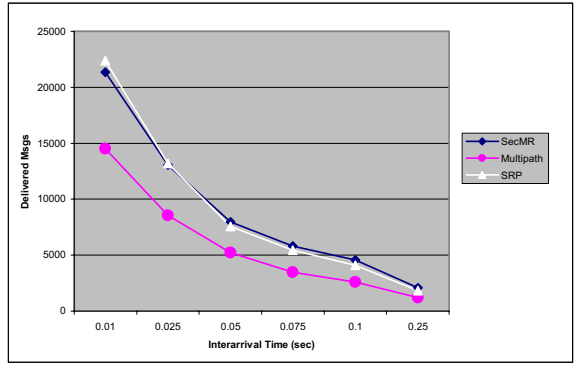

Fig. 5. Number of delivered data packet per interarrival time

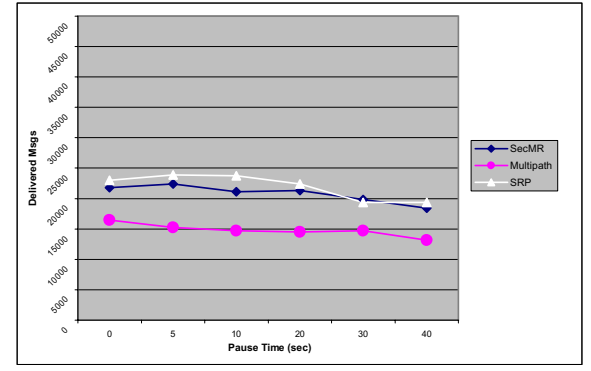

Fig. 6. Number of delivered data packet per pause time

stationary the total propagation time tends to get smaller until it reaches a minimum threshold. That does not seem to be the case for SecMR where the use of the ExcludeList prevents the request's reception by nodes that have heard it sometime in the past. In the case of SRP the protocol benefits come from the fact that each route request is forwarded only once by each intermediate node.

Figure 8 presents the average time it takes for a request message to reach its destination for the first time. If it is seen in comparison to figure 7 , it is obvious that in Multipath the request message zombies into the network for more time than in the other protocols, which causes a degradation to the network's performance. In the secure Multipath, a route request travels for a longer time than in the other two protocols, as the request is being forwarded to all nodes in range, many of which will not be included into one of the discovered paths. The route request of the SRP propagates the request towards the destination faster than the other protocols, since it rejects any variant of a specific request. The route request of the SecMR has slightly longer living times than SRP. This is reasonable as it attempts to ensure the discovery of the complete set of existing node-disjoint paths. Furthermore, the SecMR makes sure that all its neighboring nodes have contributed to the route discovery, either by participating to the RouteList (i.e. to a routing path) or by avoiding to re-process the same thread

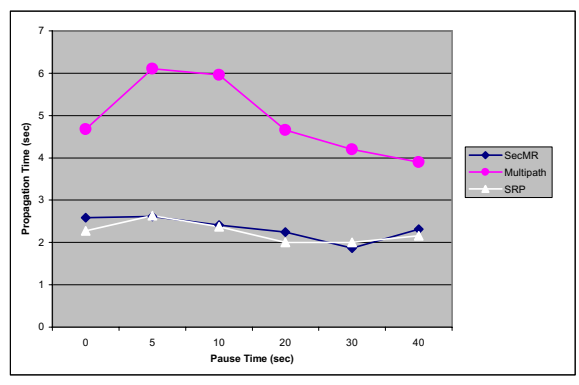

Fig. 7. Request Propagation Time per pause time

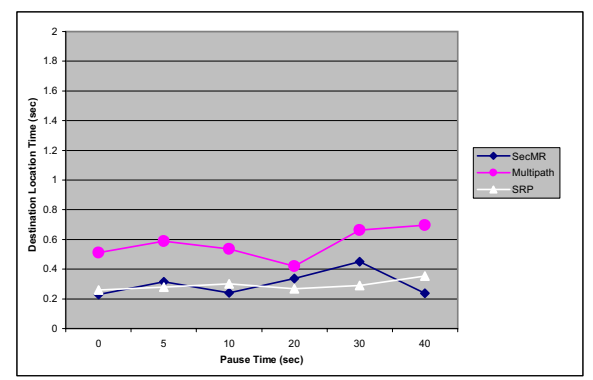

Fig. 8. Destination location time per pause time 


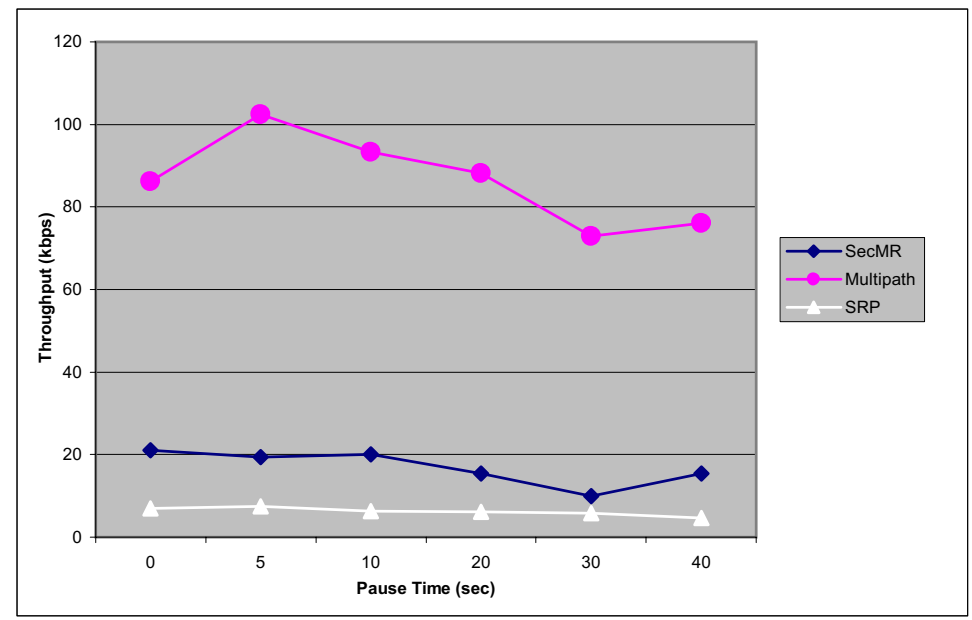

Fig. 9. Routing throughput per pause time

of the query (i.e. by participating into the ExcludeList of the query thread). The above is, also, illustrated in figure 9, which presents the total throughput derived from control messages averaged by the total number of nodes, for different pause times and with an interarrival time equal to 0.01 secs. SRP produces less control overhead than the other two protocols, thus the reduced control throughput shown in figure 9. The control throughput of SecMR is slightly increased due to the selective forwarding of request messages that it performs. Multipath has the worst performance in comparison with the other two protocols, something that is due to the number of forwards that it performs.

\section{Discussion and Future Work}

The area of ad hoc networking has received increased attention among researchers in recent years, as the evolution of wireless networking and mobile computing hardware made possible the service of various applications by this type of networks. Security in such environments is a critical issue. Over the past years a variety of new routing protocols have been proposed targeted specifically at the area of secure ad hoc networking, but little performance information and extensive comparisons between these protocols is available.

With this work we intended to examine the routing performance of three secure multipath routing protocols, namely SecMR [4], SRP [8] and Multipath [2], through various traffic conditions and under different mobility patterns. First we briefly examined their security characteristics and studied the security issues that these protocols address. Secondly, the protocols' performance was evaluated under different traffic conditions and mobility patterns.

The simulation results provide significant evidence about the efficiency of the examined secure multipath routing protocols. Our study showed that SRP 
performs better than the other two protocols, SecMR follows in short distance, while Multipath seems to be the heavier.

Considering the security characteristics (as analyzed in [4]) one can say that, Multipath achieves to provide maximum resilience against DoS attacks of collaborating malicious nodes. It provides completeness in the route discovery process and it explicitly authenticates all the intermediate nodes in each routing path. These features make it appropriate for security critical ad hoc network applications, but its applicability can only be considered in networks with relatively low density. In such environments the risk that of the request information will become larger than the message's length is minimized. Furthermore, congestion and long delays will be avoided.

The SecMR protocol also achieves completeness and provides implicit authentication of the intermediate nodes, since node authentication is performed once for a discrete time period. These features seem to make it appropriate for networks that require high security protection and present medium mobility as well as a rather high node density. In such situations, the SecMR protocol has comparable efficiency with the SRP, while it offers an increased security level.

Finally, the SRP protocol does not provide the complete set of node-disjoint paths, and it provides only end-to-end authentication. Its better routing performance makes it a suitable choice for several network configurations with increased node density. This is caused by the fact that the route request propagation avoids discovery of all the possible routes that each node could participate and in this way it converges faster. This however leads to a non-complete route discovery [4] and reduces the security resilience of the protocol to distributed DoS attacks. Thus, SRP seems suitable for applications with medium security risks.

Regarding possible extensions of our work, we consider examining the behavior of the secure multipath routing protocols in various insecure network configurations.

\section{References}

1. M. Burmester and Y. Desmedt, Secure communication in an unknown network using certificates, Advances in Cryptology - Asiacrypt '99, Lecture Notes in Computer Science Vol. 1716, Springer, 1999, pp. 274-287.

2. M. Burmester and T. van Le, Secure multipath communication in mobile ad hoc networks, Proceedings of the International Conference on Information Technology: Coding and Computing (ITCC 2004) (Las Vegas), IEEE, April 2004.

3. G. Koh, D. Oh, and Heekyoung Woo, A graph-based approach to compute multiple paths in mobile ad hoc networks, Lecture Notes in Computer Science Vol.2713, Springer, 2003, pp. 3201-3205.

4. P. Kotzanikolaou, R. Mavropodi, and C. Douligeris, Secure multipath routing for mobile ad hoc networks, Proceedings of the WONSS'05 Conference (St. Moritz, Switzerland), IEEE, January 19-21 2005, pp. 89-96.

5. S.-Ju Lee and M. Gerla, Split multipath routing with maximally disjoint paths in ad hoc networks, Proceedings of ICC 2001 (Helsinki, Finland), IEEE, June 2001, pp. 3201-3205. 
6. M. K. Marina and Samir R. Das, Ad hoc on-demand multipath distance vector routing, ACM SIGMOBILE Mobile Computing and Communications Review 6 (2002), no. 3 .

7. A. Nasipuri and S.R. Das, On-demand multipath routing for mobile ad hoc networks, Proceedings of IEEE INFOCOM99, 1999, pp. 64-70.

8. P. Papadimitratos and Z. Haas, Secure routing for mobile ad hoc networks, In Proceedings of the SCS Communication Networks and Distributed Systems Modeling and Simulation Conference (CNDS) (TX, San Antonio), January 2002.

9. A. P. Subramanian, A. J. Anto, J. Vasudevan, and P. Narayanasamy, Multipath power sensitive routing protocol for mobile ad hoc networks, Lecture Notes in Computer Science Vol.2928, Springer, 2003, pp. 171-183.

10. A. Tsirigos and Z.J. Haas, Multipath routing in the presence of frequent topological changes, IEEE Communications Magazine 39 (2001), no. 11, 132-138.

11. J. Wu, An extended dynamic source routing scheme in ad hoc wireless networks, Telecommunication Systems 22 (2003), no. 1-4, 61-75.

12. D. Johnson and D. Maltz, Dynamic source routing in ad-hoc wireless networks, Mobile Computing, Kluwer Academic Publishers, 1996, pp. 152-181.

13. Z.J Haas and M. Perlman, The performance of query control schemes for zone routing protocol, Proc. of SIGCOMM'98, 1998.

14. C. Perkins, E. Royer, and S. Das, Ad hoc on-demand distance vector routing, Proc. of IEEE Workshop on Mobile Computing Systems and Applications, IEEE, February 1999, pp. 90-100.

15. S. Marti, T.J. Giuli, K. Lai, and M. Baker, Mitigating routing misbehavior in mobile ad hoc networks, Proc. of the 6th MobiCom Conference, ACM, August 2000.

16. H. Yang, X. Meng, and S. Lu, Self-organized network-layer security in mobile ad hoc networks, Proc. of the ACM workshop on Wireless security (Atlanta, GA), ACM, September 2002, pp. 11-20.

17. S. Yi, P. Naldurg, and R. Kravets, Security-aware ad-hoc routing for wireless networks, Technical Report, UIUCDCS-R-2001-2241, June 2001.

18. L. Buttyan and J.P. Hubaux, Enforcing service availability in mobile ad hoc wANs, Proc. of the 1st MobiHoc Conference (BA, Massachusetts), August 2000.

19. J. R. Douceur, The sybil attack, In First International Workshop on Peer-to-Peer Systems (IPTPS '02), American Mathematical Society, March 2002. 